\title{
Can Left Pleural Effusion Be an Indicator of Gastric Leaks after Laparoscopic Sleeve Gastrectomy?
}

\author{
Sultan R. Alharbi \\ Department of Radiology and Medical Imaging, College of Medicine, King Saud University, Riyadh, Saudi Arabia \\ Email: drsultan000@gmail.com
}

How to cite this paper: Alharbi, S.R. (2021) Can Left Pleural Effusion Be an Indicator of Gastric Leaks after Laparoscopic Sleeve Gastrectomy? Open Journal of Radiology, 11, 11-18.

https://doi.org/10.4236/ojrad.2021.111002

Received: December 29, 2020

Accepted: February 27, 2021

Published: March 2, 2021

Copyright $\odot 2021$ by author(s) and Scientific Research Publishing Inc. This work is licensed under the Creative Commons Attribution International License (CC BY 4.0).

http://creativecommons.org/licenses/by/4.0/

\section{(c) (i) Open Access}

\begin{abstract}
Background: Coincidental pleural effusion has been observed by computed tomography (CT) scans in individual patients with gastric leaks after laparoscopic sleeve gastrectomy; however, the frequency of this phenomenon has not been investigated in cohort studies. Objectives: This study aimed to assess the diagnostic accuracy of left pleural effusion as an indicator of gastric leaks after laparoscopic sleeve gastrectomy. Setting: University hospital and bariatric surgery center of excellence. Methods: This single-center retrospective analysis included consecutive patients who had undergone laparoscopic sleeve gastrectomy followed by CT scans to investigate suspected gastric leaks from September 2011 to September 2018. The sensitivity, specificity, Youden's index, and predictive values were estimated using a $2 \times 2$ cross-tabulation. Results: The study involved assessing the CT scans of 148 patients; 80 patients (44 men and 36 women, mean age: 34 years, mean body mass index $\{\mathrm{BMI}\}: 46 \mathrm{~kg} / \mathrm{m}^{2}$ ) had positive findings of gastric leaks after laparoscopic sleeve gastrectomy, such as contrast leak, gas leak, and peri-gastric collection (either singly or in combination). The CT findings were negative for gastric leaks in 68 patients ( 38 men and 30 women, mean age: 33 years, mean BMI: $45 \mathrm{~kg} / \mathrm{m}^{2}$ ). Pleural effusion manifested as a fluid density in the dependent portion of pleural cavity on CT. The sensitivity of left pleural effusion to predict gastric leaks after laparoscopic sleeve gastrectomy was $73.8 \%$, and the specificity was $91.2 \%$. The positive and negative predictive values were $90.8 \%$ and $74.7 \%$, respectively, and the Youden's index was $64.9 \%$. Conclusion: Left pleural effusion exhibits good diagnostic accuracy for gastric leaks after laparoscopic sleeve gastrectomy. Notably, the specificity (91.2\%) was very high.
\end{abstract}

\section{Keywords}

Computed Tomography, Gastric Leak, Laparoscopic Sleeve Gastrectomy, 
Pleural Effusion

\section{Introduction}

Obesity is a worldwide pandemic, and it has a large impact on healthcare services [1] [2]. Bariatric surgery is an effective treatment for long-term weight reduction in patients with morbid obesity [3] [4] [5]. Laparoscopic sleeve gastrectomy is currently the most common bariatric surgery [6]. However, gastric leak after laparoscopic sleeve gastrectomy is a concerning potential complication, which is challenging to diagnose and treat [7]. This complication occurs in $1 \%-3 \%$ of patients [8]. Pleural effusion is another potential complication of bariatric surgery, and has a reported incidence rate of $2.63 \%$ [9].

There are few reports described pleural effusion as a thoracic complication of bariatric surgery or as a coincidental finding in post sleeve gastrectomy gastric leak [9] [10] [11].

To the best of our knowledge, there is no study to assess associations between left pleural effusion and gastric leaks after laparoscopic sleeve gastrectomy.

We hypothesized that left pleural effusion could be a diagnostic factor for gastric leaks after laparoscopic sleeve gastrectomy and investigated the associations between left pleural effusion and gastric leaks in patients who had undergone laparoscopic sleeve gastrectomy.

\section{Material and Method}

\subsection{Study Design}

This retrospective study included 161 consecutive patients who underwent abdominal computed tomography (CT) examinations after laparoscopic sleeve gastrectomy for suspected gastric leaks at a bariatric surgery center of excellence from September 2011 to September 2018. Patients presented within 1 - 3 weeks after undergoing surgery with variable clinical signs and symptoms of gastric leakage. Institutional review board approval was obtained for this study. The requirement for obtaining informed consent was waived due to the retrospective nature of this study and the use of anonymized patient data.

\subsection{Inclusion and Exclusion Criteria}

All adult patients with suspected post sleeve gastrectomy gastric leaks who underwent CT scans during the study period were included. We exclude those who underwent invasive procedures (surgery, surgical drain, percutaneous drain, or endoscopic stent or drain) prior to undergoing CT scanning for the management of post sleeve gastrectomy gastric leaks.

\subsection{CT Protocol}

Abdominal CT scans were performed using approximately $60 \mathrm{~mL}$ water-soluble 
oral contrast agent and $80-100 \mathrm{~mL}$ intravenous contrast agent. The CT acquisition was performed in the porto-venous phase 70 seconds after intravenous contrast injection, with a slice thickness of $2 \mathrm{~mm}$. Coronal and sagittal reformatted images and axial images were generated and assessed. Patients with positive findings on the CT scans were assigned to the gastric leak group, and those with negative findings were assigned to the non-leak group. All CT scans derived from patients were evaluated for left pleural effusion in the included images of the lower chest (Figure 1).

\subsection{Statistical Analysis}

Chi-square and t-tests were conducted to compare the gender, age, and body mass index (BMI) of the two groups. We also examined the ability of left pleural effusion to predict gastric leaks, using CT examination. The sensitivity, specificity, Youden's Index, and predictive values were estimated using a $2 \times 2$ crosstabulation. Asymmetric confidence intervals were also computed. The results were interpreted to determine the extent to which the diagnosis of left pleural effusion using CT examination exhibits good diagnostic accuracy for gastric leaks after laparoscopic sleeve gastrectomy.

\section{Results}

A total of 161 patients underwent CT examination for suspected gastric leaks during the study period; of these, 13 patients were excluded because they underwent previous interventions ( 4 patients with endoscopic stents, 3 patients with surgical drains, 3 patients with image-guided drains, 2 patients with chest drains, and 1 patient who underwent surgical repair of a gastric leak) for post-sleeve gastrectomy leaks. Finally, 148 patients were included in the study. The mean age of the patients was 34.9 years [ $95 \%$ confidence interval $(\mathrm{CI})=33.1$, 36.7], and their mean BMI was $45.4 \mathrm{~kg} / \mathrm{m}^{2}$ [95\% CI $\left.=44.9,45.9\right]$. Positive findings for

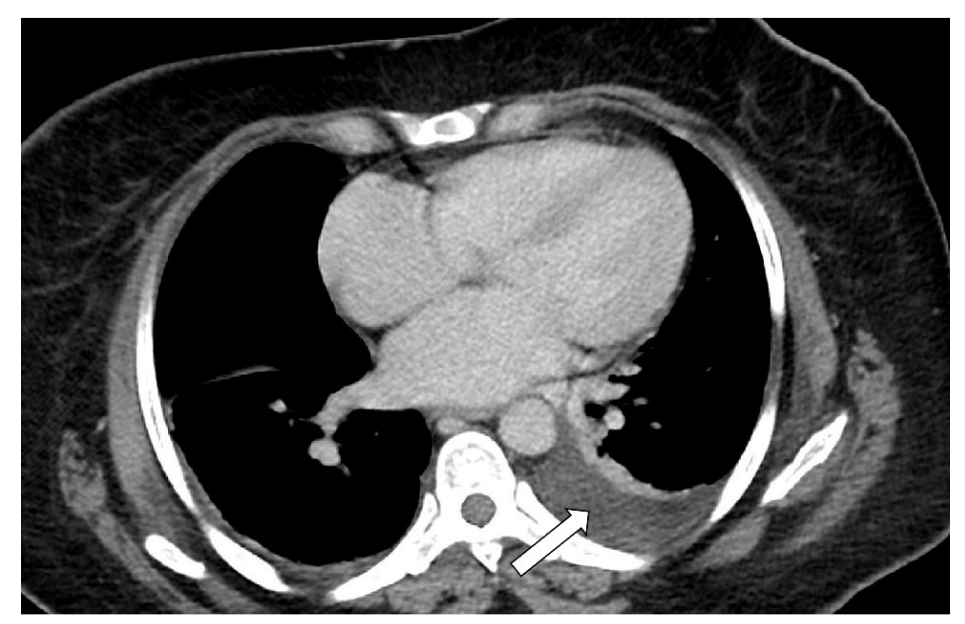

Figure 1. Axial computed tomography scan of the lower chest showing fluid density at the dependent portion of the left pleural cavity, which indicates left pleural effusion (white arrow). 
gastric leaks were observed on the CT scans in 80 patients (gastric leak group); of these, 44 were men, and 36, women. Their mean age was 34 years and mean BMI was $46 \mathrm{~kg} / \mathrm{m}^{2}$. The CT scans of the remaining 68 patients showed negative findings for gastric leaks (non-leak group). Of these 68 patients, 38 were men and 30 were women. Their mean age was 33 years and mean BMI was $45 \mathrm{~kg} / \mathrm{cm}^{2}$.

There were no significant demographic differences between the two groups. A contingency table test indicated that gastric leaks were not significantly associated with gender $\left(\chi^{2}=0.002 ; \mathrm{p}=0.964\right)$. Independent sample $\mathrm{t}$-tests indicated that age $(\mathrm{t}=0.205, \mathrm{p}=0.804)$ and BMI $(\mathrm{t}=1.04, \mathrm{p}=0.301)$ were also not significantly different between the two groups (Table 1 ).

Left pleural effusion was detected in 59 of 80 patients $(74.8 \%)$ in the gastric leak group and 6 of 68 patients $(8.8 \%)$ in the non-leak group. Left subphrenic collection was observed in 53 of 59 (89.8\%) of patients in the gastric leak group with pleural effusion (Figure 2). Four patients exhibited large amounts of loculated pleural effusion with gas pockets (Figure 3). Of the 6 patients who developed pleural effusion without gastric leaks, 5 (83\%) had other abdominal complications following sleeve gastrectomy, including 2 gastric pouch hematomas

Table 1. Baseline patient demographic data.

\begin{tabular}{cccccc}
\hline Variable & \multicolumn{2}{c}{ Non-leak group } & \multicolumn{2}{c}{ Gastric leak group } & P value \\
\hline Gender (female) & \multicolumn{2}{c}{$68(30)$} & \multicolumn{2}{c}{$80(35)$} & 0.964 \\
\hline & Mean & $95 \% \mathrm{CI}$ & Mean & $95 \% \mathrm{CI}$ & \\
Age (years) & 35.1 & $32.3,37.9$ & 34.7 & $32.3,37.1$ & 0.804 \\
BMI (kg/ms $\left.{ }^{2}\right)$ & 45.1 & $44.4,46.0$ & 45.6 & $44.9,46.3$ & 0.301 \\
\hline
\end{tabular}

CI: confidence interval, BMI: body mass index, $\mathrm{P}$ value is significant if is $<0.05$.



Figure 2. Axial computed tomography scan showing mild left pleural effusion (black arrow) associated with a gastric leak, which is evidenced by a large sub-diaphragmatic collection of air or fluid (white arrow). 


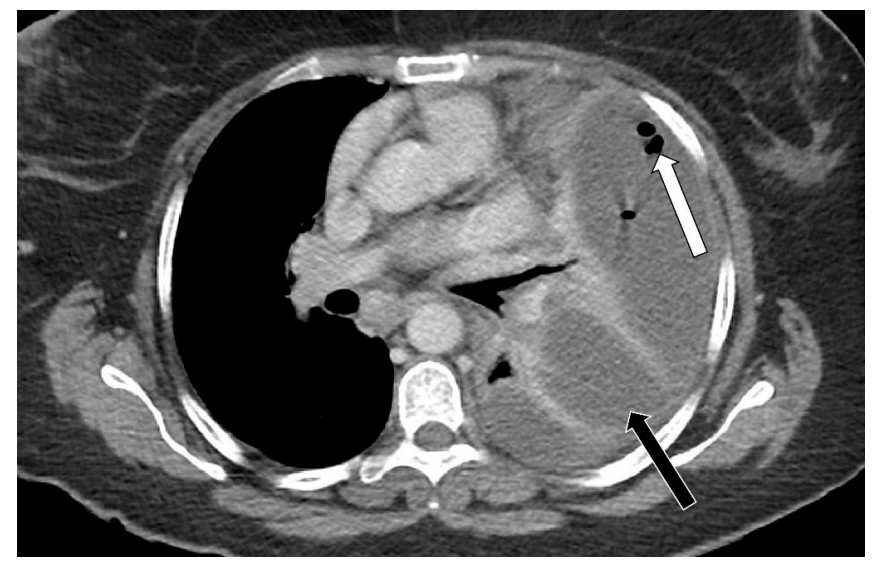

Figure 3. Axial computed tomography scan showing large multi-loculated pleural effusion (black arrow) with multiple gas pockets (white arrow).

and 3 splenic infarctions. All patients with positive findings of gastric leaks on CT scans were treated conservatively and underwent endoscopic stenting for proximal leaks identified during endoscopy. No esophageal injuries or perforations occurred in any patient.

In our study cohort, we found that the sensitivity of left pleural effusion to predict gastric leaks after laparoscopic sleeve gastrectomy was $73.8 \%$, and the specificity was $91.2 \%$. The positive and negative predictive values were $90.8 \%$ and $74.7 \%$, respectively, and the Youden's index was $64.9 \%$ (Table 2).

\section{Discussion}

Post sleeve gastrectomy gastric leak is challenging to diagnose due to variable clinical presentations and laboratory findings. Debate is still existing regarding timing and modality of imaging to diagnose post sleeve gastrectomy leak. Misdiagnosis and delayed management can lead patient morbidity and mortality. Whereas early recognition and treatment lead to better patient outcome [7] [10]. To the best of our knowledge, the present study is the first to assess the diagnostic accuracy of left pleural effusion to predict gastric leaks after laparoscopic sleeve gastrectomy.

Pleural effusion has been reported to occur after abdominal surgery at an incidence of $8 \%$; higher rates have been observed after upper abdominal procedures due to the proximity of the surgical area to the chest [12]. Postsurgical pleural effusion can be secondary to thoracic or abdominal complications, or both, e.g., esophageal perforation or peritoneal fluid collection from gastric or anastomotic leaks [13]. Other common abdominal causes of pleural effusion include subdiaphragmatic abscesses, pancreatitis, biliary tract infections, and bowel anastomotic leaks [14]. However, the pathogenesis of pleural effusions secondary to abdominal pathology is poorly understood. According to Majid et al., the proposed mechanisms include the passage of fluid through the transdiaphragmatic lymphatics or defects caused by abdominal surgery, inflammation of the vascularized muscular portion of the diaphragm, and lung atelectasis [15]. 
Table 2. Analysis of diagnostic value of pleural effusion for gastric leak.

\begin{tabular}{ccc}
\hline & Estimate & $95 \%$ CI \\
\hline Sensitivity & $73.8 \%$ & $62.5 \%, 82.7 \%$ \\
Specificity & $91.2 \%$ & $81.1 \%, 96.4 \%$ \\
Youden's Index (Sensitivity + Specificity - 1) & $64.9 \%$ & $50.3 \%, 74.1 \%$ \\
Positive Predictive Value & $90.8 \%$ & $82.5 \%, 95.9 \%$ \\
Negative Predictive Value & $74.7 \%$ & $63.7 \%, 83.3 \%$ \\
\hline
\end{tabular}

CI: confidence interval.

Gastric leaks after sleeve gastrectomy occur most commonly at the proximal gastric pouch just below the gastroesophageal junction, specifically at the angle of His. Leaks are commonly associated with adjacent left subphrenic collection [16] [17]. Examination by CT is considered to be a gold standard for diagnosing post-sleeve gastrectomy complications, including gastric leaks [6] [18]. Patients with gastric leaks after sleeve gastrectomy usually present with systemic signs of inflammation, peritonitis, abscess, and pulmonary symptoms. Pulmonary symptoms can be caused by a subphrenic abscess, empyema, or bronchogastric fistula [19]. However, gastric leakage after sleeve gastrectomy can also cause infection in the thoracic cavity, leading to loculated pleural effusion, empyema, gastropleural fistula, and lung abscess. The spread of infection into the pleural space can be visualized on CT scans as a loculated pleural effusion with pleural thickening and enhancement, with or without internal foci of air [20]. CT scans can reveal pleural effusion that cannot be seen on conventional chest radiography; further, these can also facilitate definite diagnoses and identify potential causes [21] [22].

Aljehani, et al. reported that pleural effusion occurred in $76.9 \%$ post-bariatric surgery patients which is the most encountered thoracic complication, particularly post-sleeve gastrectomy surgery [11]. AlZoubi, et al. reported that pleural effusion occurred in $52.5 \%$ of post sleeve gastrectomy gastric leak patients [12] In our cohort, left pleural effusion was detected in 64 of 65 (98\%) patients with post sleeve gastrectomy complications including gastric leak, splenic infarction or gastric pouch hematoma. Patients with gastric leak developed left pleural effusion in 59 of $80(74.8 \%)$ and most commonly associated with subphrenic collection in 53 of $59(89.8 \%)$.

The present study included some limitations. Firstly, the study was retrospective in nature, and secondly, most of the patients were referred from other centers. All our patients were stable enough to undergo CT scanning and were managed conservatively. Critically sick patients are managed surgically in our institution and were not included in our study.

\section{Conclusion}

Left pleural effusion has good diagnostic accuracy with respect to gastric leaks after laparoscopic sleeve gastrectomy, with a specificity of $91 \%$ in patients with 
suspected gastric leaks. Further prospective studies evaluating left pleural effusion as an indicator for gastric leaks based on CT scanning or chest radiography are needed to validate this result.

\section{Acknowledgements}

I would like to thank Editage (http://www.editage.com/) for English language editing.

\section{Conflicts of Interest}

The author declares that he has no conflicts of interest concerning this article.

\section{References}

[1] Deitel, M., Crosby, R.D. and Gagner, M. (2008) The First International Consensus Summit for Sleeve Gastrectomy (SG), New York City, October 25-27, 2007. Obesity Surgery, 18, 487-496. https://doi.org/10.1007/s11695-008-9471-5

[2] Treadwell, J.R., Sun, F. and Schoelles, K. (2008) Systematic Review and Meta-Analysis of Bariatric Surgery for Pediatric Obesity. Annals of Surgery, 248, 763-776. https://doi.org/10.1097/SLA.0b013e31818702f4

[3] Christou, N.V., Sampalis, J.S., Liberman, M., Look, D., Auger, S., McLean, A.P.H., et al. (2004) Surgery Decreases Long-Term Mortality, Morbidity, and Health Care Use in Morbidly Obese Patients. Annals of Surgery, 240, 416-423. https://doi.org/10.1097/01.sla.0000137343.63376.19

[4] Himpens, J., Dobbeleir, J. and Peeters, G. (2010) Long-Term Results of Laparoscopic Sleeve Gastrectomy for Obesity. Annals of Surgery, 252, 319-324. https://doi.org/10.1097/SLA.0b013e3181e90b31

[5] Buchwald, H. (2005) The Future of Bariatric Surgery. Obesity Surgery, 15, 598-605. https://doi.org/10.1381/0960892053923761

[6] Aurora, A.R., Khaitan, L. and Saber, A.A. (2012) Sleeve Gastrectomy and the Risk of Leak: A Systematic Analysis of 4,888 Patients. Surgical Endoscopy, 26, 1509-1515. https://doi.org/10.1007/s00464-011-2085-3

[7] Donatelli, G., Dumont, J.L., Cereatti, F., Ferretti, S., Vergeau, B.M., Tuszynski, T., et al. (2015) Treatment of Leaks Following Sleeve Gastrectomy by Endoscopic Internal Drainage (EID). Obesity Surgery, 25, 1293-1301. https://doi.org/10.1007/s11695-015-1675-x

[8] Currò, G., Piscitelli, G., Lazzara, C., Komaei, I., Fortugno, A., et al. (2017) Laparoscopic Sleeve Gastrectomy for Morbid Obesity: Role of Intraluminal and Intraperitoneal Postoperative Drainage. Il Giornale di Chirurgia, 38, 181-184. https://doi.org/10.11138/gchir/2017.38.4.181

[9] Abu Arab, W.S. and Alqannas, M.H. (2019) Thoracic Complications in Bariatric Surgery Patients. Asian Cardiovascular and Thoracic Annals, 27, 573-583. https://doi.org/10.1177/0218492319870926

[10] Al Zoubi, M., Khidir, N. and Bashah, M. (2020) Challenges in the Diagnosis of Leak after Sleeve Gastrectomy: Clinical Presentation, Laboratory, and Radiological Findings. Obesity Surgery, 31, 612-616. https://doi.org/10.1007/s11695-020-05008-y

[11] Aljehani, Y., AlQattan, A.S., Alkuwaiti, F.A., Alsaif, F., Aldossari, I. and Elbawab, H. (2019) Thoracic Complications of Bariatric Surgeries: Overlooked Entities. $O b$ esity Surgery, 29, 2485-2491. https://doi.org/10.1007/s11695-019-03868-7 
[12] Light, R.W. and George, R.B. (1976) Incidence and Significance of Pleural Effusion after Abdominal Surgery. Chest, 69, 621-625. https://doi.org/10.1378/chest.69.5.621

[13] Cobanoglu, U. (2011) Pleural Effusion Resultant after Upper Abdominal Surgery: Analysis of 47 Cases. Journal of Clinical and Analytical Medicine, 2, 16-20. https://doi.org/10.4328/JCAM.305

[14] Nielsen, P.H., Jepsen, S.B. and Olsen, A.D. (1989) Postoperative Pleural Effusion Following Upper Abdominal Surgery. Chest, 96, 1133-1135. https://doi.org/10.1378/chest.96.5.1133

[15] Majid, A., Ochoa, S., Chatterji, S., Fernandez-Bussy, S., Kheir, F., Rivera, E., et al. (2017) Safety and Efficacy of Tissue Plasminogen Activator and Dnase for Complicated Pleural Effusions Secondary to Abdominal Pathology. Annals of the American Thoracic Society, 14, 342-346. https://doi.org/10.1513/AnnalsATS.201608-594BC

[16] Casella, G., Soricelli, E., Rizzello, M., Trentino, P., Fiocca, F., Fantini, A., et al. (2009) Nonsurgical Treatment of Staple Line Leaks after Laparoscopic Sleeve Gastrectomy. Obesity Surgery, 19, 821-826. https://doi.org/10.1007/s11695-009-9840-8

[17] Alharbi, S.R. (2020) Percutaneous CT-Guided Drainage of Gastric Leaks Post-Sleeve Gastrectomy. Open Journal of Radiology, 10, 1-8. https://doi.org/10.4236/ojrad.2020.101001

[18] Alharbi, S.R. (2020) Computed Tomography-Based Diagnosis of Post-Laparoscopic Sleeve Gastrectomy Gastric Leak. Journal of Clinical Imaging Science, 10, 8. https://doi.org/10.25259/JCIS_5_2020

[19] Pequignot, A., Fuks, D., Verhaeghe, P., Dhahri, A., Brehant, O., Bartoli, E., et al. (2012) Is There a Place for Pigtail Drains in the Management of Gastric Leaks after Laparoscopic Sleeve Gastrectomy? Obesity Surgery, 22, 712-720.

[20] Galgano, S.J., Sonavane, S.K., Sanyal, R., Singh, S.P., Menias, C.O. and Bhalla, S. (2017) Thoracic Imaging after Bariatric Surgery. Journal of Thoracic Imaging, 32, W45-W53. https://doi.org/10.1097/RTI.0000000000000286

[21] Arenas-Jiménez, J., Alonso-Charterina, S., Sánchez-Payá, J., Fernández-Latorre, F., Gil-Sánchez, S. and Lloret-Llorens, M. (2000) Evaluation of CT Findings for Diagnosis of Pleural Effusions. European Radiology, 10, 681-690. https://doi.org/10.1007/s003300050984

[22] Jany, B. and Welte, T. (2019) Pleural Effusion in Adults-Etiology, Diagnosis, and Treatment. Deutsches Ärzteblatt International, 116, 377-386. https://doi.org/10.3238/arztebl.2019.0377 